\title{
CORRELACIÓN ENTRE GROSOR CORNEAL CENTRAL, PRESIÓN INTRAOCULAR Y AFECTACIÓN GLAUCOMATOSA DEL CAMPO VISUAL
}

\author{
RELATIONSHIP BETWEEN CENTRAL CORNEAL THICKNESS, \\ INTRAOCULAR PRESSURE AND SEVERITY OF GLAUCOMATOUS \\ VISUAL FIELD LOSS
}

\author{
JIMÉNEZ-RODRÍGUEZ E ${ }^{1}$, LÓPEZ-DE-COBOS $\mathrm{M}^{1}$, LUQUE-ARANDA R ${ }^{2}$, \\ LÓPEZ-EGEA-BUENO MA², VÁZQUEZ-SALVI AI², GARCÍA-CAMPOS JM ${ }^{3}$
}

\section{RESUMEN}

Objetivo: Determinar el grosor corneal central (GCC) en sujetos normales, pacientes glaucomatosos e hipertensos oculares, valorando si el GCC está asociado con la presencia de glaucoma o de hipertensión ocular. Por otra parte, comprobar la correlación entre el GCC y el estadio de afectación del campo visual (CV).

Método: Se realizó un estudio de casos y controles, con 150 ojos de 150 pacientes, divididos en tres grupos: normales (47 ojos), hipertensos oculares (35) y glaucomatosos (68). A su vez, se establecieron tres subgrupos dentro de los sujetos glaucomatosos, dependiendo de la afectación del CV según la clasificación de Hodapp de defectos del campo visual (inicial, moderada y avanzada). Se valoró la campimetría, la medida del GCC mediante paquimetría ultrasónica y de la PIO con tonómetro de aplanación de Goldmman (TAG). Se aplicó el test de hipótesis t-Student para muestras independientes que siguen una distribución normal.

Resultados: La media del GCC en los pacientes glaucomatosos era 526 micras Desviación Estándar

\begin{abstract}
Objective: to determine central corneal thickness in normal subjects, glaucomatous patients and ocular hypertension patients, to evaluate if the central corneal thickness is related to the presence of glaucoma or ocular hypertension. Furthermore, we aim to verify the relationship between central corneal thickness and severity of glaucomatous visual field loss.

Methods: comparative study including 150 eyes of 150 subjects, separated into three groups: normal (47 eyes), ocular hypertension (35) and glaucomatous (68). This last group was subdividided in three subgroups depending on the Hodapp-ParrishAnderson criteria for scoring Humphrey visual field defects (initial, moderate and advanced). We evaluated the visual field, the central corneal thickness measured by pachymetry and the intraocular pressure measured by Goldmann tonometry. Results were analysed using the Student's t-test for normally distributed independent samples.

Results: the central corneal thickness was $526 \pm 25$ microns (mean \pm standard deviation (SD)) in glaucomatous patients, $560 \pm 27$ microns in ocular
\end{abstract}

\footnotetext{
Recibido: 28/11/08. Aceptado: 27/2/09.

Servicio de Oftalmología. Hospital Clínico de Málaga. Málaga. España.

${ }^{1}$ Licenciado en Medicina.

2 Doctor en Medicina.

3 Doctor en Medicina. Jefe del Servicio.

Correspondencia:

Encarnación Jiménez Rodríguez

C/. Antonio Trueba, 14 , Blq. $5,9^{\circ} 2$

29017 Málaga

España

E-mail: ejimrod@yahoo.es
} 
(DE) 25, 560 DE 27 en hipertensos oculares y 556 DE 27 en los sujetos normales, con diferencias significativas entre el grupo de pacientes glaucomatosos y los otros dos $(p<0,01)$, pero no entre el grupo de hipertensos oculares y el de sujetos normales $(\mathrm{p}=0,45)$. En los sujetos glaucomatosos, se encontraron diferencias con significación estadística entre el GCC del subgrupo con afectación avanzada del CV y los de afectación inicial y moderada $(\mathrm{p}<0,01)$, pero no entre estos dos últimos $(\mathrm{p}=0,7)$. Conclusiones: El GCC en pacientes glaucomatosos es menor que en los sujetos controles e hipertensos oculares. Los pacientes glaucomatosos con pérdida de CV avanzada cursan con córneas más delgadas que aquéllos con alteración campimétrica inicial o moderada.

Palabras clave: Grosor corneal central, paquimetría, campo visual, glaucoma, hipertensión ocular. hypertension patients, and $556 \pm 27$ microns in the normal group. Statistical significance could be found between the glaucomatous group of patients and the other groups $(p<0.01)$, but not between the ocular hypertension group and normal subjects $(\mathrm{p}=$ 0.4 ). The comparison between central corneal thickness of the subgroup with advanced damage of the visual field and the two other subgroups was also statistically significant ( $\mathrm{p}<0.01)$, but not between the subgroups of initial damage and moderate damage $(\mathrm{p}=0.7)$.

Conclusions: the central corneal thickness in glaucomatous patients is lower than in normal subjects and in ocular hypertension patients. Patients classified as having advanced damage in their visual field have significantly lower central corneal thickness measurements than patients classified as having initial or moderate damage (Arch Soc Esp Oftalmol 2009; 84: 139-144).

Key words: central corneal thickness, pachymetry, visual field, glaucoma, ocular hypertension.

\section{INTRODUCCIÓN}

La presión intraocular (PIO) es uno de los parámetros más importantes en el diagnóstico y tratamiento del glaucoma. El tonómetro de aplanación de Goldmann (TAG) es aún el método «gold standard» para su medición $(1,2)$. Diversos estudios han demostrado una correlación lineal positiva entre la PIO y el GCC determinado mediante paquimetría, sugiriéndose que existiría una subestimación de la PIO en las córneas delgadas y una sobreestimación en las gruesas $(1,3)$. El OHTS (Ocular Hypertension Treatment Study) definió las córneas delgadas como factor de riesgo en sujetos hipertensos oculares para el desarrollo de glaucoma manifiesto (4). Herdon et at describieron el GCC como un factor clínico importante a tener en cuenta en la determinación de la severidad del glaucoma en el examen inicial del especialista (5).

Por otra parte, el estudio del campo visual (CV) continúa siendo hoy día la prueba prínceps para el diagnóstico de la enfermedad glaucomatosa, e incluso antes de introducir cualquier nuevo método diagnóstico para esta patología es necesario validarlo con el CV (2).

El propósito de este estudio es determinar el GCC medio en sujetos normales de nuestra pobla- ción, y compararlo con el de pacientes glaucomatosos e hipertensos oculares. Además, pretende estudiar la correlación existente entre las medidas paquimétricas y el estadio de afectación del campo visual de los sujetos diagnosticados de glaucoma, y así valorar la paquimetría como posible factor de riesgo independiente en la progresión de la enfermedad glaucomatosa.

\section{SUJETOS, MATERIAL Y MÉTODOS}

Se llevó a cabo un estudio de casos y controles en el que se incluyeron 150 ojos de 150 pacientes, con edades comprendidas entre los 25 y 78 años, procedentes del servicio de Oftalmología de nuestro centro.

Se excluyeron del estudio aquellos pacientes con edema corneal, queratoplastia, cirugía previa de catarata, ptisis bulbi o anormalidades morfológicas del disco óptico (papilas grandes y laterovertidas).

En cada paciente se valoraban los resultados de la última perimetría automatizada realizada en los seis meses anteriores mediante un campímetro Humphrey Field Analyzer modelo 745 (estrategia 24-2 SITA standard), siendo requisito indispensable que ésta cumpliera los criterios de fiabilidad (6) y que no se 
tratase del primer CV del paciente, el GCC expresado en micras y medido con el paquímetro ultrasónico Pacline (Optikon 2000, Roma, Italia) (tomando como valor real la media de cinco mediciones realizadas por el mismo explorador), la toma de la PIO con el TAG y la excavación papilar evaluada bajo midriasis farmacológica con lente de no contacto de 90D. Se establecieron tres grupos dentro del estudio:

1. Grupo de sujetos controles (47 ojos): sin patología oftalmológica evidente, con PIO $\leq 21 \mathrm{mmHg}$ y sin lesión en la perimetría ni en el examen de la cabeza del nervio óptico.

2. Grupo de hipertensos oculares (35 ojos): con $\mathrm{PIO}>21 \mathrm{mmHg}$, sin alteraciones campimétricas ni en el disco óptico.

3. Grupo de glaucomatosos (68 ojos): con PIO controlada farmacológica y/o quirúrgicamente, así como con lesión en la campimetría y/o cambios morfológicos de la papila sugestivos de glaucoma. Los pacientes de este grupo fueron subdivididos a su vez en tres subgrupos dependiendo del grado de afectación del CV según la clasificación de Hodapp (7):

a) Inicial (18 ojos).

b) Moderada (22 ojos).

c) Avanzada (28 ojos).

Para el análisis estadístico se utilizaron los programas informáticos R y SPSS (SPSS Inc, Illinois, USA). Se aplicó el test de hipótesis t-Student para muestras independientes que siguen una distribución normal. Los resultados se consideraron estadísticamente significativos cuando la $\mathrm{p}<0,05$.

Se asumió que el GCC presenta una distribución normal, ya que se trata de una variable continua muestreada de una población natural. La validez de dicha hipótesis de normalidad se verificó mediante la comparación, por medio de gráficas tipo «q-q plot», de valores medidos del GCC con valores generados aleatoriamente que siguen una distribución normal.

Mediante el análisis estadístico t-Student realizado se verificó si existían diferencias significativas desde un punto de vista estadístico en el GCC de cada uno de los grupos considerados: sujetos control, pacientes glaucomatosos e hipertensos oculares. Asimismo se empleó el análisis t-Student para establecer si existían diferencias estadísticamente significativas en el GCC en cada subgrupo considerado dentro del grupo de pacientes glaucomatosos.

\section{RESULTADOS}

150 ojos de 150 pacientes fueron incluidos en el análisis. De ellos, 47 (31,33\%) ojos pertenecían al grupo control, $35(23,33 \%)$ al grupo de los sujetos hipertensos oculares y por último $68(45,33 \%)$ al de los glaucomatosos, de los que $18(26,47 \%)$ tenían afectación inicial del CV, 22 (32,35\%) afectación moderada y $28(41,17 \%)$ afectación avanzada.

La media del grosor corneal central fue de 556 micras DE 27 en el grupo de sujetos de normales, 560 DE 27 en el grupo de hipertensos y 526 DE 25 en el de glaucomatosos (tabla I). Respecto a la diferencia de medias entre los tres grupos, fue significativa entre el grupo de glaucomatosos y el de hipertensos oculares $(34 ; \mathrm{p}<0,01)$, y entre los glaucomatosos y los sujetos normales $(30 ; \mathrm{p}<0,01)$, pero no entre el grupo de hipertensos y el de casos controles $(-4,5 ; p=0,45)$ (fig. 1$)$.

En lo referente a los subgrupos de los ojos glaucomatosos según la afectación del $\mathrm{CV}$, la media del GCC en los ojos con afectación inicial del CV fue de 532 micras DE 28, 534 DE 22 en los casos de afectación moderada del CV y 517 DE 22 en los de afectación avanzada (tabla II). La diferencia de medias entre los pacientes con alteración campimétrica inicial y moderada no fue significativa $(-2$ micras; $\mathrm{p}=0,7)$, pero sí lo fue entre los pacientes con daño perimétrico inicial y avanzado (17 micras; $\mathrm{p}<0,05)$ y los de afectación del CV moderada y avanzada (13 micras; $\mathrm{p}<0,01)$ (fig. 2).

\section{DISCUSIÓN}

Nuestros resultados sugieren que el GCC es significativamente más grueso en el grupo control y en el de sujetos hipertensos oculares que en el grupo de pacientes glaucomatosos. Estos resultados son concordantes con la bibliografía revisada (1-5,8-10). Por otra parte, según nuestro estudio, el GCC tam-

Tabla I. Media del GCC, desviación típica y error típico de la media en el grupo de sujetos controles, HTO y glaucomatosos

\begin{tabular}{lccc}
\hline & $\begin{array}{c}\text { Media } \\
\text { GCC }\end{array}$ & $\begin{array}{c}\text { Desviación } \\
\text { típica }\end{array}$ & $\begin{array}{c}\text { Error típico } \\
\text { de la media }\end{array}$ \\
\hline Controles & 556 & 27 & 3,94 \\
HTO & 560 & 27 & 4,6 \\
Glaucomatosos & 526 & 25 & 3,04 \\
\hline \hline
\end{tabular}




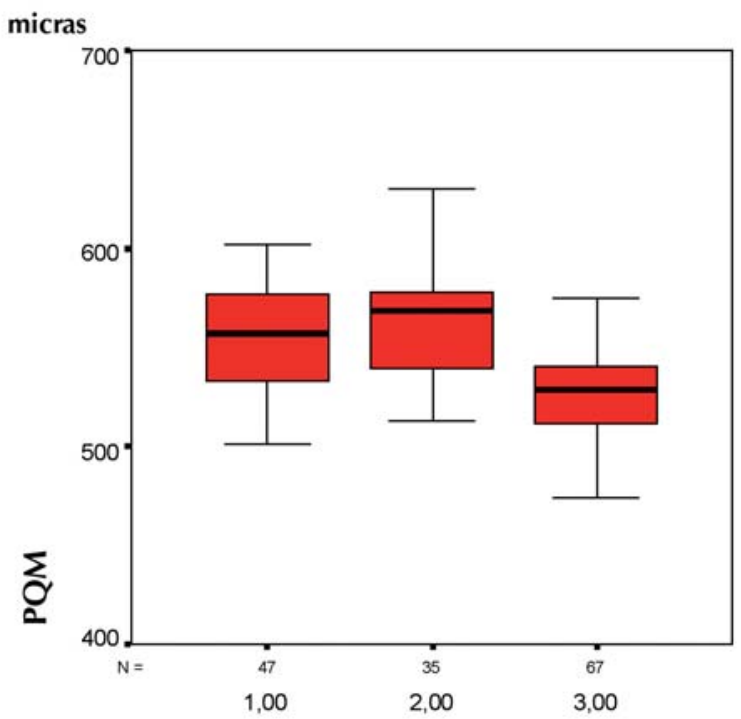

DIAG

Fig. 1: Gráfico tipo «caja y bigotes» que muestra la diferencia de medias del GCC en cada uno de los tres grupo incluidos en el estudio: (1) sujetos controles, (2) hipertensos oculares, (3) pacientes glaucomatosos. Los bordes de la caja representan el primer y tercer cuartil del conjunto de datos, y la línea horizontal que atraviesa la caja representa la mediana. Los bigotes se extienden hasta el valor más extremo que no esté a una distancia de más de 1,5 veces el rango intercuartil desde el extremo de la caja.

bién estaría relacionado con el estadio de afectación del CV, existiendo una asociación estadísticamente significativa entre ambas variables; no hemos encontrado un acuerdo unánime respecto a este punto en los estudios publicados. Jonas et al. (11), Sullivan-Mee et al. (12) y ensayos como el «Early Manifest Glaucoma Trial (EMGT)» (13) coinciden en que el GCC no se puede considerar como un factor de riesgo significativo para la progresión del glaucoma, sin embargo Herndon et. al. (5), Medei-

Tabla II. Media del GCC, desviación típica y error típico de la media según la afectación inicial, moderada 0 avanzada del $\mathrm{CV}$

\begin{tabular}{lccc}
\hline & $\begin{array}{c}\text { Media } \\
\text { GCC }\end{array}$ & $\begin{array}{c}\text { Desviación } \\
\text { típica }\end{array}$ & $\begin{array}{c}\text { Error típico } \\
\text { de la media }\end{array}$ \\
\hline Afectación inicial CV & 532 & 28 & 6,70 \\
Afectación moderada CV & 534 & 22 & 4,78 \\
Afectación avanzada CV & 517 & 22 & 4,22 \\
\hline \hline
\end{tabular}

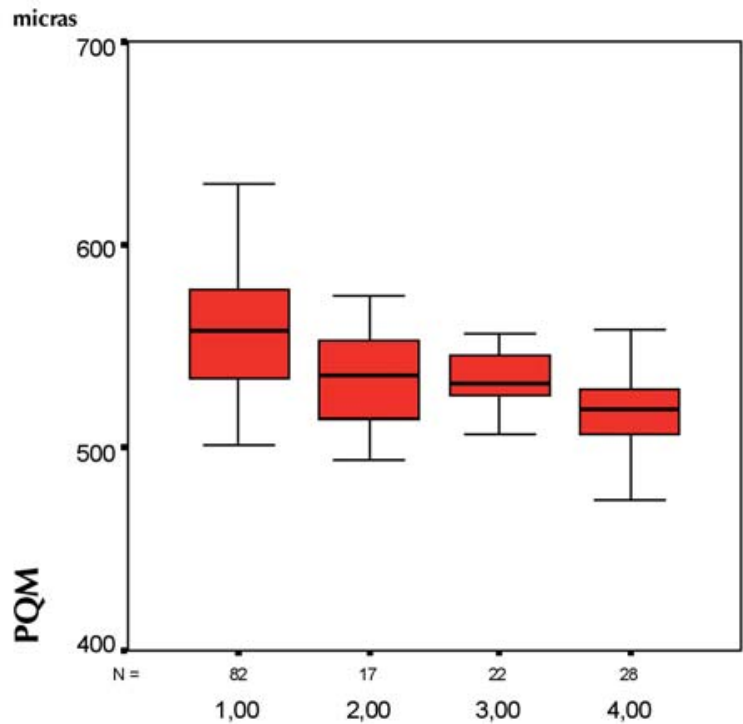

\section{CV}

Fig. 2: Gráfico tipo «caja y bigotes» que muestra la diferencia de medias del GCC según la afectación del CV: (1) sin afectación, (2) afectación inicial, (3) afectación moderada, (4) afectación avanzada. Los bordes de la caja representan el primer y tercer cuartil del conjunto de datos, y la línea horizontal que atraviesa la caja representa la mediana. Los bigotes se extienden hasta el valor más extremo que no esté a una distancia de más de 1,5 veces el rango intercuartil desde el extremo de la caja.

ros et. al. (9), «The Ocular Hypertension Treatment Study (OHTS)» (4) y el mismo Sullivan-Mee et al. en publicaciones posteriores (14) apoyan nuestros resultados, encontrando el GCC delgado como un factor de riesgo predictivo de la pérdida de CV.

No están muy claros los motivos de estos resultados tan discordantes. Podrían ser debidos a los diferentes diseños de cada estudio (diferencias en las poblaciones incluidas en cada uno y en los criterios de definición de pérdida o afectación de CV, así como el tratamiento empleado en cada estudio).

La demostración de que el GCC en sujetos hipertensos oculares y en los casos controles es mayor que la de los pacientes glaucomatosos es sugestiva de que las córneas gruesas serían protectoras frente al daño glaucomatoso, y viceversa, las córneas delgadas tendrían un mayor riesgo de progresión de daño $(3,15,16)$.

En conclusión, nuestros resultados demuestran que el GCC en pacientes glaucomatosos es signifi- 
cativamente menor que en sujetos normales o hipertensos oculares. Además encontramos una relación estadísticamente significativa entre el GCC y el CV, en el sentido de que existe mayor pérdida campimétrica en los pacientes con menor GCC. Esto último sugiere la importancia de cuantificar el GCC en sujetos glaucomatosos, ya que si éste es bajo habría que plantear una PIO objetivo menor que si el GCC fuera normal $(1,3-5,8,9,11)$.

\section{BIBLIOGRAFÍA}

1. Kniestedt C, Lin S, Choe J, Nee M, Bostrom A, Sturmer J, et al. Correlation between intraocular pressure, central corneal thickness, stage of glaucoma, and demographic patient data: prospective analysis of biophysical parameters in tertiary glaucoma. J Glaucoma 2006; 15: 91-97.

2. Miglior S, Guareschi M, Romanazzi F, Albe E, Torri V, Orzalesi $N$. The impact of definition of primary openangle glaucoma on the cross-sectional assessment of diagnostic validity of Heidelberg retinal tomography. Am J Ophthalmol 2005; 139: 878-887.

3. Doughty MJ, Zaman ML. Human corneal thickness and its impact on intraocular pressure measures: a review and metaanalysis approach. Surv Ophthalmol 2000; 44: 367- 408.

4. Gordon MO, Beiser JA, Brandt JD, Heuer DK, Higginbotham EJ, Johnson CA, et al. The Ocular Hypertension Treatment Study. Arch Ophthalmol 2002; 120: 714-720.

5. Herndon LW, Weizer JS, Stinnett SS. Central corneal thickness as a risk factor for advanced glaucoma damage. Arch Ophthalmol 2004; 122: 17-21.

6. Alward W. Glaucoma. Los requisitos en oftalmología. Madrid: Ediciones Harcourt 2001; 70-72.

7. Hodapp E, Parrish RK, Anderson DR. Initial treatment of primary open-angle glaucoma. In: Hodapp E, Parrish RK,
Anderson DR. Clinical decisions in glaucoma. St. Louis: Mosby: 1993; 52-59.

8. Singh RP, Goldberg I, Graham SL, Sharma A, Mohsin M. Central corneal thickness, tonometry, and ocular dimensions in glaucoma and ocular hypertension. J Glaucoma 2001; 10: 206-210.

9. Medeiros FA, Sample PA, Zangwill LM, Bowd C, Aihara $M$, Weinreb RN. Corneal thickness as a risk factor for visual field loss in patients with preperimetric glaucomatous optic neuropathy. Am J Ophthalmol 2003; 136: 805813.

10. Weizer JS, Stinnett S, Herndon LW. Longitudinal changes in central corneal thickness and their relation to glaucoma status: an 8 year follow up study. Br J Ophthalmol 2006; 90: 732-736.

11. Jonas JB, Stroux A, Velten I, Juenemann A, Martus P, Budde WM. Central corneal thickness correlated with glaucoma damage and rate of progression. Invest Ophthalmol Vis Sci 2005; 46: 1269-1274.

12. Sullivan-Mee M, Halverson KD, Saxon GB, Saxon MC, Qualls C. Relationship between central corneal thickness and severity of glaucomatous visual field loss in a primary care population. Optometry 2006; 77: 40-46.

13. Leske MC, Heijl A, Hussein M, Bengtsson B, Hyman L, Komaroff E, et al. Factors for glaucoma progression and the effect of treatment: the early manifest glaucoma trial. Arch Ophthalmol 2003; 121(1): 48-56.

14. Sullivan-Mee M, Gentry JM, Qualls C. Relationship between asymmetric central corneal thickness and glaucomatous visual field loss within the same patient. Optom Vis Sci 2006; 83: 516-519.

15. Shimmyo M, Ross AJ, Moy A, Mostafavi R. Intraocular pressure, Goldmann applanation tension, corneal thickness, and corneal curvature in Caucasians, Asians, Hispanics, and African Americans. Am J Ophthalmol 2003; 136: 603-613.

16. Brubaker RF. Tonometry and corneal thickness. Arch Ophthalmol 1997; 117: 104-105. 
